\title{
Wireless Technologies in Healthcare: Selected Papers from IEEE PIMRC 2011
}

\author{
Kamran Sayrafian-Pour · Kamya Yekeh Yazdandoost
}

Received: 2 August 2012/ Accepted: 2 August 2012/Published online: 10 August 2012

(C) Springer Science+Business Media, LLC (outside the USA) 2012

\begin{abstract}
The use of wireless technologies for medical device communication, health monitoring (at hospitals or homes) and mobile healthcare information delivery (i.e. $\mathrm{m}$-Health) is one of the most rapidly growing areas in health-IT research today. The papers which appear in this special issue have been carefully selected from the best IEEE PIMRC 2011 conference. They are highlighting various challenging issues in using wireless technology for healthcare applications such as PHY \& MAC innovations for wearable and implantable medical sensors, optical communication and location systems in hospital environments and interference mitigation issues.
\end{abstract}

Keywords Body area networks - Mobile health technology $\cdot$ Wireless health monitoring

The use of wireless technologies for medical device communication, health monitoring (at hospitals or homes) and mobile healthcare information delivery (i.e. m-Health) is one of the most rapidly growing areas in health-IT research today. More and more healthcare organizations are embracing wireless technology, to reduce cost and at the same time improve care. By eliminating wired connections to deliver medical information and increasing mobility of the patients and healthcare professionals, treatment

\footnotetext{
K. Sayrafian-Pour $(\square)$

National Institute of Standards and Technology,

Gaithersburg, MD, USA

e-mail: kamran.sayrafian@nist.gov; ksayrafian@nist.gov

K. Yekeh Yazdandoost

National Institute of Information and Communications

Technology, Tokyo, Japan

e-mail: yazdandoost@nict.go.jp
}

outcomes will undoubtedly improve. Advances in novel micro-electronics design and manufacturing can also increase the value of wireless technology in improving healthcare service productivity and efficiency.

Integration of appropriate wireless technologies with the existing information infrastructure will create a truly pervasive environment for many critical healthcare applications. This will not only have a great impact on costefficient delivery of some healthcare services but ultimately will lead to improving the quality of life for many individuals. The vast number of attractive and cutting edge applications in this cross-disciplinary field can revolutionize healthcare processes.

The papers which appear in this special issue have been carefully selected from the best IEEE PIMRC 2011 conference papers addressing some of the challenging issues related to the wireless technologies in healthcare. The invited authors have been asked to provide a significantly extended version of the respective conference paper, which has subsequently undergone a rigorous review process according to the IJWIN publication standards prior to acceptance. The final set of papers addresses various challenging issues in using wireless technology for healthcare applications such as PHY \& MAC innovations for wearable and implantable medical sensors, optical communication and location systems in hospital environment, and interference mitigation issues. Our objective is to give the reader a perspective on current technical achievements and also future development in these exciting areas. We sincerely hope you enjoy reading the interesting and high-quality research works presented here.

The authors of the first paper "RTS-MAC: A Relative Time Synchronization MAC Protocol for Low Duty Cycle Body Sensor Networks" address energy efficiency in some medical applications of body sensor networks. Specifically, 
an artificial Accommodation System which is a set of two small active medical implants aiming at restoring accommodation of the human eye is discussed in the paper. The authors "Christoph Beck, Jörg Nagel, Peter Hevesi, and Georg Bretthauer" present a relative time synchronization medium access control protocol (RTS-MAC), which keeps relative time synchronization between two or more sensor nodes in a very energy-efficient manner. RTS-MAC makes use of the periodic broadcast of regular data messages and exploits the inter-arrival times to predict future arrivals within tight boundaries. Thereby, no overhead is generated for synchronization purposes, and still, idle listening is reduced to a minimum, which solely depends on the shortterm accuracy of the underlying clock systems. The proposed protocol has been implemented using off-the-shelf components and employed the internal low-accuracy oscillators of the microcontrollers as clock sources. Therefore, a very low duty cycles close to an ideal minimum has been achieved. Further, the results indicate that through omitting external crystals in favor of a minimally larger battery, the battery life of a sensor node can be prolonged.

The second paper "Empirical Performance of RSSIBased Monte Carlo Localization for Active RFID Patient Tracking Systems" by William Cully, Simon Cotton, and William Scanlon presents a RSSI-based Monte Carlo Localization that was implemented using commercial $868 \mathrm{MHz}$ off-the-shelf hardware. Many of the proposed localization systems overlook the unique radio environment presented by the human body leading to systematic errors and inaccuracies when deployed in real life situations. The empirical data that has been gathered across a relatively large number of scenarios within a single indoor office environment shows that the body shadowing effect caused by the human body introduces path skew into location estimates. It has been shown that, by using two body-worn nodes in concert, the effect of body shadowing can be mitigated by averaging the estimated position of the two nodes worn on either side of the body.

Interference could be a significant challenge for wireless Body Area Networks (BANs). This important issue has been addressed in the third paper "IEEE 802.15.6 DPSK Impulse-Radio Ultra-Wideband Physical Layer: Receiver Architectures and Interference Performance" by Igor Dotlic and Ryu Miura. Performances of the two lowest data rates of Impulse-Radio Ultra-Wideband DPSK physical layer of the recently published IEEE 802.15.6 BANs standard is analyzed in this paper. Two receiver architectures suitable for the reception of symbols with signal structure described in specification of this physical layer, namely duty-cycled sampling receiver and chirp receiver, have been introduced. Then, performance of these receiver architectures have been analyzed through probabilities of error in different phases of packet reception. Analysis has been performed in the presence of different types of interference; namely Frequency Modulated Ultra-Wideband, WiMax and other co-located IEEE 802.15.6 ImpulseRadio Ultra-Wideband devices.

An approach to maintain reliable operation in the presence of interference for BANs is using adaptive schemes. This is the topic of the fourth paper "Interference Mitigation using Adaptive Schemes in BANs" by Wen-Bin Yang and Kamran Sayrafian-Pour. Since practical implementation of power control mechanisms could be very challenging in BANs, link adaptation schemes can be an efficient alternative to preserve link quality while allowing more number of nodes to operate simultaneously. The authors in this paper propose several interference mitigation schemes such as adaptive modulation as well as adaptive data rate and duty cycle. The concept of Interference Mitigation Factor has been introduced as a measure to quantify the effectiveness of the proposed schemes. The cited adaptive schemes are relatively simple and well-suited for low power nodes in BANs that might be operating in environments with high level of interference.

The fifth paper "Transmission Power Analysis of Optical Wireless based Mobile Healthcare Systems" by Seyed Sina Torkestani, Nicolas Barbot, Stephanie Sahuguede, Anne Julien-Vergonjanne, and Jean-Pierre Cances considers the problem of using infrared (IR) communication for the transmission of information in hospital environments. In this paper, the authors have considered a system where the patient can move within a hospital room and the data collected by sensors are transmitted to a distant receiver placed on the ceiling. Considering two main IR propagation types (line of sight and diffuse), the IR mobile channel has been characterized by using a random waypoint mobility for a realistic scenario. The authors then evaluate the outage probability of this mobile monitoring scheme. From the results, the power efficiency of IR technology to ensure the communication between sensors and the collecting point has been addressed. Also, to maximize the system reliability, the possibility of using channel coding has been investigated. The analysis shows that for a typical data rate of $500 \mathrm{Kbps}$, it is theoretically possible to minimize the transmission power considering the use of a forward error correction code while maintaining the targeted information rate.

The sixth paper "Radiation Pattern of Wearable Antennas: A Statistical Analysis of the Influence of the Human Body" by Michal Mackowiak, Carla Oliveira and Luis M. Correia explores a statistical approach for modeling antennas' behavior in the vicinity of the human body. The coupling between the body and the antenna, and the reduction of antenna efficiency lead to a distortion of the radiation pattern, which depends on the distance as well as 
the location on the body. In this paper, the statistics of radiation patterns have been calculated for Uniform and Rayleigh antenna-to-body distance Distributions. Results show that the relative change of the average radiation pattern for an antenna located on the chest can reach $24 \%$. The study has been complemented with measurements, showing that, in the area of interest for on-body communications, an average difference between CST and measurements is about $0.9 \mathrm{~dB}$.

The human body is a crucial part of the transmission medium in BANs. The seventh paper "Characteristic and Modeling of Human Body Motions for BAN Applications" by Ruijun Fu, Yunxing Ye, and Kaveh Pahlavan derives a statistical model for communication links in the Medical Implant Communication Service band, Industrial Scientific Medical band and Ultra-Wideband (UWB) based on the narrowband measurement. Second order statistics, including level crossing rate and fade duration have been presented in order to evaluate the link quality and outage performance for on-body to on-body communication scenarios. Using a network analyzer, Doppler spread spectrum in frequency domain and coherence time from temporal variations of human body movements have also been analyzed from different perspectives. The proposed on-body to on-body channel model for human body motions can be used to better design wireless network protocols for BANs.

"Accuracy of RSS-based RF localization in multicapsule endoscopy" is the topic of the eighth paper which has been addressed by Yunxing Ye, Pranay Swar, Kaveh Pahlavan and Kaveh Ghaboosi. In this paper, authors derive and analyze cooperative localization bounds for endoscopic wireless capsule as it passes through the human gastrointestinal (GI) tract. Using a three dimensional human body model and log-normal models for RSS propagation from implants to body surface, the Cramer-Rao bounds (CRB) of location estimators have been calculated for the three segments of the human digestive system: stomach, small intestine and large intestine. Authors have also provided analysis of the factors affecting localization accuracy, including various organ environments; external sensor array topology, number of pills in cooperation and the random variations in transmit power of sensor nodes. The simulation results show that the number of receiver sensors on body surface has more influence on the accuracy of localization than the number of pills in cooperation inside the GI tract.

In conclusion, we would like to express our thanks to the authors of all papers in this special issue. Their excellent works on extending the conference version of their papers on this emerging technology will undoubtedly make this special issue a success. We also extend our gratitude to the reviewers whose meticulous reviews and thoughtful comments have enhanced the innovative ideas presented here.
Finally, we would like to sincerely thank the devoted staff of Springer for their high level of professionalism and assistance during the preparation of this special issue.

\section{Author Biographies}

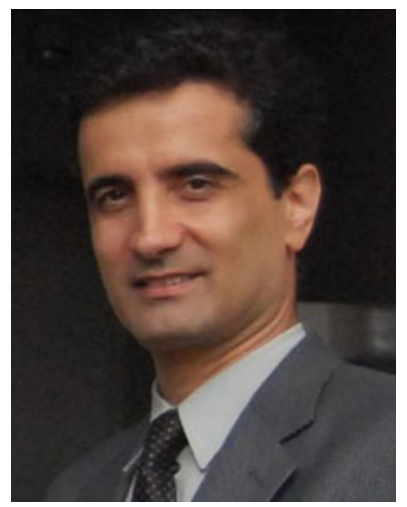

Kamran Sayrafian-Pour is a program lead at the Information Technology Laboratory of the National Institute of Standards and Technology (NIST) located in Gaithersburg, Maryland. He leads several strategic projects that are focused on Pervasive healthcare technologies. He is also an adjunct faculty of the University of Maryland. Prior to this, he was the cofounder of Zagros Networks, Inc. A fabless semiconductor company based in Rockville, Maryland where he served as President and senior member of the architecture team. He is the co-inventor/inventor of four U.S. patents. He has served as invited member of technical program committee of many international conferences. He has also been the co-chair and organizer of several strategic tracks and workshops focused on application of wireless communication in healthcare. His research interests include medical body area networks, mobile sensor networks and RF-based indoor positioning. He has published over 70 conference and journal papers, and book chapters in these areas. He was the recipient of the IEEE PIMRC 2009 \& SENSORCOMM 2011 best paper awards. He was also a contributing member and the co-editor of the channel modeling document of the IEEE802.15.6 international standardization on body area networks. Dr. Sayrafian-Pour holds Ph.D., M.S. and B.S. degrees in Electrical \& Computer Engineering from University of Maryland, Villanova University and Sharif University of Technology, respectively. He is a senior member of IEEE.

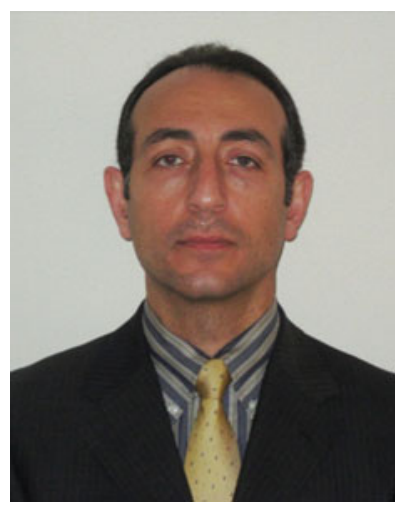

Kamya Yekeh Yazdandoost received his $\mathrm{Ph} . \mathrm{D}$. degree from University of Pune, Pune, India. He joined the Communications Research Laboratory (CRL), Japan in 2003 (now, National Institute of Information and communications Technology: NICT), and has been engaged in research on Ultra WideBand (UWB) and on Wireless Body Area Network (BAN) technology. He is currently working as a Senior Researcher at Dependable Wireless Laboratory, Wireless Network Research Institute, NICT, Japan. He is an adjunct Professor with University of Oulu, Oulu, Finland and he is a recipient of Finland Distinguished Professor Program (FIDIPRO) Fellow. He is a senior member of the IEEE (Institute of Electrical and Electronics Engineers) and life member of the IEEE Microwave Theory and Techniques Society. He was the IEEE Chair in the Committee for Channel Modeling of Body Area Network Standardization and coeditor of the channel modeling documents of the IEEE.802.15.6 on Body Area Network Standardization. He was a representative of the NICT, Japan to the European COST2100 Action (Management 
Committee Meeting on Pervasive Mobile and Ambient Wireless Communications) and currently representative to the COST-IC1004 Action and chairing the Body Environment Group of this Action. He is also a Member of European Microwave Association. He is Associate Editor of International Journal of Microwave Science and Technology. He is a co-author of the book Wireless Body Area
Network and recipient of the best paper award of the IEEE PIMRC 2009 conference. He has over 100 published papers and holding 10 patents. He was the co-chair of PIMRC2011 conference track on Wireless Networks and Health Care and has been TPC member and session chair of various international conferences. 